\title{
Il pataffio di Luigi Malerba, tra storicità e attualità. Una società in crisi e la lotta di classe
}

\section{Fawzi Mohamad Issa ${ }^{1}$}

Facoltà Al Alsun - Università di Ain Shams

Received: 18-06- 2021 Accepted: 06-07-2021 Available Online: 07 -07-2021

https://doi.org/10.36602/faj/2021.n18.02

\begin{abstract}
This study aims to analyze Luigi Malerba's novel Il pataffio, from a new perspective, namely that of its double value between historicity and actuality. Despite being set in the Middle Ages, the work reflects not only the social and political reality experienced and perceived by the writer in Italy in the sixties and seventies, but also reflects the contemporary one in other parts of the world. The novel is therefore nothing more than a denunciation of the multiform crisis of numerous contemporary societies where values are lacking, and where evil dominates in all its forms. By analyzing the plot of the work and the development of its story we will try to outline the traits of totalitarian regimes and highlight the catastrophic consequences of absolutism. The study will also highlight the similarity of the class struggle in the novel and the conditions experienced by some peoples under totalitarian regimes, especially in the countries of the Arab Spring, where the rebellion of the peoples and their revolution against the tyrants were the protagonist of the scene in the last few years.
\end{abstract}

PAROLE CHIAVE: Il pataffio - Malerba - storicità - attualità lotta di classe

fawziissa@hotmail.com ${ }^{1}$ 
رواية البتافيو للويجي ماليربا بين التاريخية والمعاصرة: أزمة الجتتمع والصراع بين الطبقات

$$
\text { د. فوزي محمد عبد الحميد عيسى الألسن - جامعة عين شمس }
$$

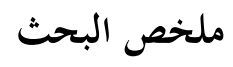

تمدف هذه الدراسة إلى تحليل رواية لويجي ماليرب| "إلبتافيو" من منظور جديد، ألا وهو القيمة المزدوجة التي بتمع بين التاريخية والمعاصرة. فعلى الرغم من وقوع أحداث الرواية في العصور الوسطى، إلا أها تعكس ليس فقط الواقع الاجتماعي والسياسي الذي عاشه وأدركه الكاتب في إيطاليا في الستينيات والسبعينيات، وإنما تعكس أيضًا الواقع المعاصر في أجزاء أخرى من العالم. وبالتالي، فإن الرواية ليست سوى إدانة للأزمة متعددة الأشكال في العديد من المجتمعات المعاصرة حيث تنعدم القيم، ويسود الشر بجميع أشكاله. ومن خلال تحليل حبكة العمل وتطور أحداثه، سنحاول تحديد سمات الأنظمة الشمولية وإبراز العواقب الكارثية للاستبداد. كما ستسلط الدراسة الضوء على تشابه الصراع الطبقي في الرواية والظروف التي تعيشها بعض الشعوب في ظل الأنظمة الشمولية، خاصة في بلدان الربيع العربي، حيث كان تمرد الشعوب وثورتم على الطغاة هو الذي يهكم المشهد في السنوات القليلة الماضية.

$$
\text { الكلمات المفتاحية: إلبنافيو - ماليربا - تاريخية - معاصرة - صراع طبقي }
$$




\section{INTRODUZIONE}

Romanziere di successo e pluripremiato, Luigi Malerba è stato oggetto di numerosi studi e ricerche, per la sua copiosa produzione letteraria. La sua scrittura dalle forme plurime ha indotto i critici letterari e i linguisti a dedicare grandi spazi allo studio e all'analisi della sua opera. Le opere di Malerba offrono numerose dimensioni per la loro ricchezza e la loro varietà, dovute alle sue numerose esperienze. Per la sua varia e distinta produzione letteraria, caratterizzata da una dimensione surreale, Malerba è stato uno degli autori italiani più tradotti del Secondo Novecento. Tra la sua vasta produzione romanzesca, Malerba scrisse tre romanzi storici: Il pataffio, 1978, di sfondo medioevale, Il fuoco greco, 1990, di sfondo bizantino, e Le maschere, 1994, di sfondo rinascimentale.

Ambientato in un Medioevo irreale e grottesco, Il pataffio ha una trama imperniata su una vicenda buffa e fantastica dove succede di tutto: il marconte Berlocchio di Cagalanza prende in sposa la contessa Bernarda di Montecacchione, una donna grassa e sgraziata, e riceve perciò in dote il fatiscente castello di Tripalle.

Dopo un lungo e faticoso viaggio in cui succede di tutto Berlocchio e il suo sgangherato seguito arrivano a destinazione e allora scoprono che il dono non è altro che una maledizione, poiché entro i confini del feudo non vi sono che lumache, bisce e scorpioni e il palazzo cade a pezzi, a causa delle intemperie e dei terremoti. I contadini del luogo l'hanno trasformato in una stalla.

Per il marconte il sogno si trasforma in incubo e il suo scontro con i villani è inevitabile. In un susseguirsi di situazioni grottesche gli abitanti del feudo, guidati da Migone, non si fanno lusingare dalle fumisterie dei sedicenti potenti, ma reagiscono con la sagacia contadina di chi ha imparato tanto dalla vita. Il marconte, con l'inganno e la manipolazione mostra la sua vera natura di despota che commette ogni tipo di crimine contro il proprio popolo, spingendolo alla rivoluzione. 


\subsection{PROBLEMATICA DELLA RICERCA}

Come molte altre opere malerbiane, Il pataffio presenta diverse chiavi di lettura, cosa che rende difficile coglierne subito il suo vero senso. Dato ciò l'opera va guardata e letta da una nuova prospettiva. Studiata e analizzata con una chiave nuova, cioè quella del suo doppio valore che unisce tra storicità $\mathrm{e}$ attualità, il romanzo può essere considerato una denuncia e un grido dello scrittore non solo contro la situazione in Italia, ma anche contro la realtà vissuta in altre parti del mondo.

Con il suo sfondo medioevale, l'opera viene classificata come un romanzo storico, e non ricorda esplicitamente la realtà contemporanea a cui rappresenta una forte allusione. Tuttavia, il lettore attento può intravvedere facilmente che dietro alla sua vicenda buffa e fantastica c'è un messaggio molto profondo e significativo.

\subsection{DOMANDE O IPOTESI}

- Quali sono gli aspetti della crisi descritta nell'opera e che legame ha con la realtà attuale?

- Perché lo scrittore usa questo stile sarcastico ed ironico?

- Perché la vicenda è ambientata nel Medioevo?

- Quali sono le caratteristiche dell'assolutismo di Berlocchio nel romanzo e quali sono i punti in comune con i regimi totalitari nei nostri giorni.

\subsection{OBIETTIVI}

Questo studio mira quindi a guardare l'opera da una nuova prospettiva e analizzarla con una chiave diversa per rivelare il suo vero messaggio celato sotto la forma storica, collegandone la vicenda alla realtà contemporanea sia in Italia, che in altre parti del mondo. Si mira anche a mettere in rilievo gli aspetti della crisi multiforme trattata nel romanzo e i punti in comune con la realtà della società contemporana.

Cercheremo anche di mettere in risalto i motivi che stanno alla base dello stile sarcastico e ironico adottato da Malerba in questa 
opera e delineare la caratteristiche dei regimi totalitari e le consegueze catastrofiche dell'assolutismo, attraverso l'analisi dello sviluppo della vicenda del romanzo.

\subsection{TERMINI DELLA RICERCA}

Il pataffio tra storicità e attualità - una società in crisi- crisi multiforme: sociale, morale e religiosa - crisi politica e lotta di classeassolutismo- regimi totalitari.

\subsection{STUDI CORRELATI}

Chiefele, Anna, Ironia: metafinzione nelle opere di Luigi Malerba, Tesi di dottorato, Graduate Department of Italian Studies, University of Toronto, 2010.

Crotti, Cecilia, "Indovina le strutture e $i$ contenuti seguiranno”, Tre studi narratologici sul romanzo di Luigi Malerba, Tesi di laurea in Letteratura italiana contemporanea, Università di Bologna, Scuola di Lettere e beni culturali, Anno accademico 20162017.

Lenci, Laura Marzia, Contesti Malerbiani, Poetica sperimentale e rappresentazione della scena matrimoniale, Università degli studi di Verona, Scuola di Dottorato di Studi Umanistici, Dipartimento di Culture e Civiltà, 2013.

\section{METODOLOGIA}

La ricerca adotta il metodo analitico-descrittivo: seguendo lo sviluppo della vicenda e analizzando il comportamento dei personaggi e le varie componenti dell'opera, cercheremo di mettere alla luce il messaggio simbolico trasmesso dallo scrittore. Attraverso l'analisi degli episodi più significativi e di alcuni dialoghi e situazioni cercheremo di dare risposta alla domande sopracitate. 


\section{TRATTAMENTO E RISULTATI}

\subsection{IL PATAFFIO TRA STORICITÀ E ATTUALITÀ}

L'opera, classificata come un romanzo storico, rispecchia chiaramente lo sperimentalismo linguistico e letterario dello scrittore. Uno degli aspetti di questo sperimentalismo consiste nel doppio valore del romanzo, poiché malgrado sia ambientata nel medioevo la vicenda può essere considerata la metafora della situazione non solo in Italia del secondo Novecento, ma anche in diverse altre parti del mondo, perciò:

In ogni caso, la storicità viene falsata sotto numerosissimi aspetti che passano in particolare dalla rappresentazione e concezione del potere, di cui si esplicita principalmente l'anacronismo: i ragionamenti di Migone di fronte all'idea di società feudale, stilizzata e stereotipata, di Berlocchio sono lo specchio di una serie di prese di coscienza politiche che appartengono alla contemporaneità, o quanto meno che identificano un passaggio di concezione storica che nasce negli ultimi secoli (Crotti, 2016-2017, p. 107).

Dato ciò, la comicità e l'umorismo dominanti nell'opera non sono altro che una maschera che cela l'indignazione e la critica dello scrittore nei confronti del sistema politico e sociale in Italia degli anni Sessanta e Settanta. Tale indignazione e tale critica possono anche estendersi verso la realtà politica e sociale alla fine del Novecento e all'inizio del terzo Millennio in tante altre parti del mondo:

Malerba riesce perfettamente a narrare il contingente e anche per tale ragione le sue opere si prestano ad essere decifrate come una metafora dell'oggi. Il presente, non ancora finito, viene vissuto come un momento storico ineffabile, i cui legami con il passato e soprattutto con il futuro non sono ancora stati identificati. Solo l'uomo del futuro, tramite un processo di intramazione, può ricomporre coerentemente il nostro presente, ovvero il suo passato, illudendosi di aver così rintracciato la verità storica (Chiafele, 2010, pp. 103-104).

Se la vicenda si svolge all'interno di un involucro storico, si tratta di un involucro dentro il quale lo scrittore ha voluto occultare le frecce 
della sua critica, lanciate contro la corruzione politica, morale e religiosa nel suo Paese. Basti pensare alla logica, al metodo e alle idee di Migone nella sua campagna di consapevolizzazione per i suoi compaesani per rendersi conto che siamo di fronte a ideali liberali tipici dell'età moderna e dell'uomo moderno e non certamente del Medioevo:

Era l'esplosione del comico malerbiano in una formulazione quasi parossistica del "ludismo formale" (M. Corti) che poteva trovar venia, da una parte, per la ribollente e spregiudicata invenzione, dall'altra, per la possibilità di leggere in controluce quesl Medioevo sullo sfondo dei diversi ma analoghi orrori dell'età nostra (Manacorda 1996, p. 673).

Lo scrittore ricorre anche all'aspetto storico come mezzo di distanziamento dalla realtà che sta raccontando, cosa che gli consente di penetrare nella percezione del lettore sensibile che, pian piano, si accorge che tra le righe c'è un'allusione alla propria realtà quotidiana: Più degli altri romanzi, quindi, le opere medievali del nostro autore sono costituite da una serie di aneddoti, tra loro poco connessi. Anche per questa ragione, ma non solo, Il pataffio, Storie dell'anno mille e I cani di Gerusalemme vengono spesso interpretati come una metafora del presente (Chiafele, 2010, p. 102).

E, come dice Mauri, P. (2013), uno scrittore contemporaneo si serve qualche volta del passato per raccontare di traverso il presente, proprio come fa in questo romanzo Malerba che usa la storia per dimostrare che il mondo non funziona e bisognerebbe guidarlo. La storia gli fa comodo e lo conforta nella sua visione delle cose (pp. 9091).

Infatti, le ingiustizie, le oppressioni e i soprusi subiti dai poveri villani tripallesi ad opera del marconte e della sua guarnigione hanno condotto, alla fine, alla rivoluzione del popolo di Tripalle contro la classe governante. Si tratta di una situazione paragonabile a quella vissuta da molti altri popoli che vengono sfruttati e oppressi da una classe governante corrotta. Una volta accortosi di questa realtà il lettore comincia inevitabilmente a riflettere sulle proprie condizioni e 
a paragonare tra la propria vita quotidiana e quella di Migone e dei villani nel romanzo. Da questo paragone potrebbe, di certo, scaturire una reazione aperta a tutti gli scenari, inclusi quelli più gravi. Migone e il suo ragionamento possono, cioè, diventare una fonte di ispirazione per molti altri, come pure il popolo di Tripalle può diventare un esempio da seguire per molti popoli che vivono le stesse condizioni sotto il governo dei tiranni.

D'altronde, si può paragonare la vicenda dell'opera con quello che è successo nello scorso decennio in molti Paesi della primavera araba dove i popoli, una volta accorti della loro realtà misera e delle ingiustizie subite, si sono ribellati contro i loro despoti. Proprio in questo aspetto consiste l'attualità de $I l$ pataffio. Non solo, ma la realtà rivelata alla fine dell'opera, cioè che Migone non è altro che un dettatore moderno, è quasi identica ai risultati della primavera araba in molti paesi dove i nuovi governi non sono che dittature moderne, più pericolose e più onerose di quelle precedenti:

Esiste, infatti, una grande differenza tra il desiderio di potere di Berlocchio e quello di Migone; il marconte Berlocchio, essere vile, meschino, stolto ed inconcludente, in realtà, non dovrebbe incutere così tanto terrore. I suoi atteggiamenti, dopo tutto, sono scontati e soprattutto prevedibili; con un po' d'astuzia e d'intelligenza è quindi possibile reagire ai suoi numerosi soprusi. Il furbo Migone, invece, è potenzialmente più pericoloso del marconte; i due personaggi rappresentano, infatti, due mentalità tra loro opposte. Berlocchio è l'uomo del passato, medievale, mentre Migone ha in sé alcune caratteristiche del dittatore moderno e per questo è da ritenersi più temibile (Chiafele, 2010, p. 103).

\subsection{UNA SOCIETÀ IN CRISI}

L'opera, da questo punto di vista, può essere considerata una denuncia di una società in crisi. Una crisi multiforme dove mancano $i$ valori, la dignità dell'uomo, la giustizia e dove dominano la miseria e il male in tutte le sue forme. Questo vuol dire che Malerba era consapevole della situazione politica, economica, religiosa e sociale 
del proprio Paese, e che il romanzo non è altro che un segno di sdegno verso tale situazione:

In primo luogo, l'autore, fortemente cosciente della crisi religiosa, morale e politica del suo tempo, sviluppa una riflessione che, partendo dall'osservazione del mondo a lui coevo, arriva alla constatazione, cinica e ironica al contempo, di vivere in una società dominata dal paradosso: il male, la crisi dei valori e l'arrivismo politico nascono con la nascita della società stessa. Proprio questa riflessione è alla base della scelta di descrivere in alcune opere realtà e avvenimenti di epoche passate (la realtà feudale del Pataffio o la decadenza della Roma rinascimentale nelle Maschere); una scelta tesa a dimostrare la perfetta coincidenza tra passato e presente (De Palma, 2012, p. 25).

Fin dalla prima pagina, l'autore mette in risalto la crisi economica e la miseria generale che fanno da sfondo per tutta l'opera, disegnando un quadro molto espressivo dove appare il corteo del marconte mentre si avvia verso il palazzo di Tripalle e il feudo donatogli dal padre della sposa. Tutto il corteo soffre la fame e la fatica del viaggio, persino i cavalli che tirano le carrozze degli sposi e di frate Capuccio. La campagna introno fa parte della tetra scena, apparendo priva di ogni segno di vita come se fosse devastata da una calamità naturale.

La scena risulta più cupa con l'ambiente circostante, poiché oltre alla fatica, la fame, e la polvere sollevata dalla strada che acceca gli occhi, ci sono anche turbe di moscherini come nuvole nel cielo che attaccano gli uomini e gli animali. Il corteo, perciò, invece di arrivare al feudo, perde la strada e si mette nei pasticci:

Il cielo è annuvolato a tratti da turbe di moscherini molestissimi che si buttano a succhiare gli occhi ai cavalli e ai soldati, già mezzo cecati per la polvere. È per via di questo cecamento generale da polvere e da moscherini che il corteo del marconte Berlocchio de Cagalanza si è sperso nella piana del Tevere (Malerba, 2015, p. 7). 
La miseria e la crisi economica risultano più chiare quando il marconte fa un giro ai confini del proprio feudo per compilare un inventario di tutti i possedimenti. Egli si rende conto della differenza tra il proprio feudo e quelli adiacenti: nel proprio feudo la campagna è magra e secca con qualche albero morto in piedi, e in quelli vicini invece verdeggiano prati e olivi, e ci sono greggi di pecore, pollami, e capannoni di animali. Allora,

Berlocchio avrebbe una gran voglia di dire sui denti a Bernarda sua moglie e al re di Montecacchione suo padre: tenerevi questo feudo de merda e io me ne vado! Dopo il viaggio di ispezione guidato dal villano Migone, Berlocchio di chiude nella sua stanza e quasi si metterebbe a piangere per la rabbia. Gli avevano promesso un feudo e invece gli hanno dato una terra di miserabilia, un castello diroccato e i sudditi più magri e più ladri del reame. Chissà quanto ride il re di Montecacchione, ma si vedrà, chi ride ultimo (Malerba, 2015, p. 111).

\subsection{CRISI MORALE E RELIGIOSA}

La crisi morale e religiosa viene riflessa dall'autore in modo stravagante attraverso tutta l'opera e fin dall'inizio, a partire dal matrimonio stesso di Berlocchio con Bernarda, figlia del re di Montecacchione. Non si tratta di un matrimonio normale, ma di un matrimonio di convenienza o di un affare, il cui unico obiettivo è quello di ottenere la dote della sposa e acquistare un titolo nobile. Si nota infatti che, fin dall'inizio lo sposo non nutre nessun affetto per la sua sposa né la minima attrazione verso di lei. Anzi, lui trova sempre le scuse per non consumare il matrimonio, mentre Bernarda, brutta e grassa come è, appare impaziente e chiede continuamente con volgarità il rapporto coniugale.

Si tratta perciò di un matrimonio emblematico con cui lo scrittore mette in rilievo la dissacrazione del matrimonio e della famiglia nelle nostre società moderne. Perciò come dice Lenci:

Lungi dal demonizzare la vita coniugale, dal sovvertirne i valori o dal rifiutarne la necessità, Luigi Malerba assume un 
atteggiamento interrogativo che gli consente di mantenere ciò che vi è di esistente, portando all'attenzione del lettore alternative, peraltro non sempre plausibili, sulle ragioni del matrimonio (Lenci, 2013, pp.2829).

La crisi morale viene espressa anche esplicitamente nella relazione carnale tra i due armigeri, Ulfredo e Manfredo, che praticano la sodomia anche durante il viaggio nella loro tenda di accampamento. Questa mancanza di moralità risulta più palese soprattutto nella reazione degli altri soldati agli atti osceni commessi dai due armigeri, una reazione partecipe come se fosse tutto normale. Questo atteggiamento potrebbe essere considerato un'allusione a quanto succede oggi in molte società dove l'omosessualità sta diventando una normalità:

Che cosa stanno facendo i due compari come tutte le notti è risaputo dai soldati che manco li ascoltano più come una musica troppo sentita. Vanno avanti a chiamarsi così per una mezza ora o poco presso e poi si sente un orlo di gola da ambedue insieme. Finalmente si indormentano quando già tutto il campo dorme nel sonno sprofondo della notte (Malerba, 2015, p. 21).

Le oscenità commesse assumono qualche volta gli aspetti più strani e più assurdi, come la relazione carnale che Berlocchio ha con la somara trovata nel bosco. Egli la conduce poi al palazzo, e mentre tutti alla corte pensavano di aver già trovato la carne deliziosa da arrostire, egli ordina di preparare una stanza nella corte per la somara e le dà il nome di Bianchetta. Non solo, ma poi chiama frate Capuccio e gli chiede di celebrare il proprio matrimonio con l'animale:

"Et quod altro fare potest de asina qua presente?"

Risponde Berlocchio con voce sicura:

"Matrimonium celebrare".

"Matrimonium infra chi? Ubi sunt contraentes?".

"Io e Bianchetta".

"Bianchetta cognoscere non cognosco".

"Bianchetta è la somara che vedere potete davanti a li occhi vostri" (Malerba, 2015, p. 136). 
La crisi morale e religiosa è rispecchiata soprattutto dalla condotta di frate Capuccio che, invece di essere il custode dei valori supremi della religione, si comporta come una persona buffa e ipocrita. Egli commette tutti i peccati che proibisce ai suoi parrocchiani. Se non in realtà, li commette in sogno, perché secondo lui "sognare il peccato non è peccato". E allora di notte si abbandona ai suoi sogni pieni di lussuria e peccati carnali. Ha una donna tutta sua in quei sogni, una donna che si chiama Adelaide che gli torna quasi tutte le notti, e qualche volta lo incontra anche durante il sonnellino che fa nel pomeriggio. Quasi sempre Adelaide appare in camicia, ma alcune volte si presenta già nuda e gli fa commettere tutti i peccati possibili (Malerba, 2015, pp. 44-46).

D'altronde, il prete non solo asseconda il marconte nei suoi decreti arbitrari contro i sudditi e le sue voglie oscene, ma anche favoreggia le sue porcherie. Per esempio, credendo che il villano Migone sta per morire in seguito alle quarantotto frustate ricevette, egli si reca nella casa del villano con un soldato e cerca di portare via con la forza la moglie di Migone, Margherita, per condurla dal Marconte che la vuole possedere. E quando trova che il marito della donna è vivo e sta migliorando, cerca di ucciderlo con due colpi in testa dati da lui e dal suo aiutante.

L'ipocrisia di frate Capuccio risulta molto chiara quando Berlocchio gli chiede di celebrare nella parrocchia il proprio matrimonio con al somara Bianchetta. Il prete, anche se non gli dà il suo consenso al matrimonio considerandolo una bigamia, gli consiglia di avere relazione con essa e far finta di niente (Malerba, 2015, p. 138).

Non solo ma commette anche l'adulterio, tenendo una relazione segreta con la moglie del marconte, Bernarda, che trova in lui la propria soddisfazione sessuale che il marito non le dà. E così mentre Berlocchio tiene una relazione carnale con la somara, la marcontessa lo tradisce con il prete:

Si sente l'ansimare del galoppo ancora per un po'. Migone resta poggiato al muro nel buio fino a quando viene un urlo da tutte $\mathrm{e}$ 
due le voci, una specie di muggito furioso che fa tremare i muri. Migone ha recognosciuto le voci di Bernarda e di frato Capuccio, che adesso sbuffano stremati dalla fatica. Si ritira in punta di piede per ritornare nella stanzetta da dove era venuto mentre si risente la voce ansimante del frato (Malerba, 2015, p. 168).

Il culmine della miseria e della crisi morale e religiosa appare poi nell'episodio in cui Baldassarre er Faticone, un villano tripallese, decide di vendere al mercato del villaggio sua moglie Cisira perché non è più in grado di mantenerla né di soddisfarla sessualmente. Dopo essere diventato allo stremo delle forze e senza un soldo come tutti gli altri suoi compaesani, questo villano si approfitta della soldataglia arrivata al paese con il marconte per mettere in vendita la moglie come prevede una antica disposizione capitolare e costumanza del reame di Montecacchione:

Badassarre er Faticone monta su un panchetto e incomincia:

“Sordati! Ce sta n'usanza delli tempi der nonno der mi' nonno, quanno che li omeni se faceveno respetta' da la moje sia drento la casa che da fora. L'usanza era cusì confatta che un marito quanno che nun je garbava più di vive co' 'na moje che j'aveva mancato de respetto, sia pe' raggione de corna che pe' raggione de cattiveria o de antro, ciaveva er deritto de menarla ar mercato come se mena 'na vaccina, e qui la metteva in vendita ar mejo offerente. Adunque ciavete davante a li occhi la moje mia che ho deciso de vende con il suo propio regittimo consenso. [...]" (Malerba, 2015, p. 91).

Davanti a questa scena gli altri villani si raggruppavano, si guardavano in faccia e si ridevano nelle orecchie. Baldassere, che aveva fissato il prezzo della moglie con sette baiocchi, sapeva che nessuno di loro possiede nemmeno un baiocco, perciò mirava invece ai soldati arrivati di recente nel paese. In verità anche i soldati vivevano la miseria, perciò sette di loro si mettono a parlare fra loro $\mathrm{e}$ decidono di comprare la moglie di Baldassarre in società e di goderla tutti insieme: ognuno la tiene per un giorno alla settimana.

Questo episodio può essere considerato il più emblematico e il più rappresentativo della dissacrazione della famiglia e del legame 
coniugale, un fenomeno che si sta diffondendo nelle società di oggi. Riflette anche la misera e la crisi generale e soprattutto la mancanza di valori morali e religiosi in una società in cui la donna si è trasformata in una merce che si vende al mercato e uno strumento di pochissimo valore per soddisfare il desiderio maschile, perciò come dice Renato Barilli (2013):

Ecco perché, in lui, comicità e sessualità vanno sempre a braccetto, e si deve anche aggiungere che l'impertinenza di tanti sberleffi e offese recate al senso comune del pudore, a tutti i principi autoritari, è anche un modo per dare sfogo a uno spirito aggressivo, portato a una rivolta, che però non riesce o non vuole prendere la via dell'effettualità, ma si accontenta di gesti di profanazione e di oltraggio mantenuti a un livello simbolico, come minacce, come digrignamento dei denti, più che come atti fisicamente consistenti ( $\mathrm{p}$. $83)$.

\subsection{CRISI POLITICA E LOTTA DI CLASSE}

Come succede tuttora nei paesi governati da regimi totalitari, dove il potere viene esercitato da una sola persona, la miseria e la crisi economica conducono, alla fine, alla ribellione del popolo e allo scontro con il regime. È quello che dice la vicenda de Il pataffio dove i tripallesi si ribellano contro l'ingiustizia e l'assolutismo del marconte. Lo scontro si trasforma in una accanita tra governati e governanti. Tale lotta è dovuta soprattutto allo sfruttamento, ai soprusi ed ai tributi che spingono la popolazione all'estremo.

Alla base della rivoluzione è stato anche il carattere stesso del marconte e la sua natura di despota che prende le decisioni più arbitrarie e spietate. In principio, appena arrivato al feudo decide di prendere possesso delle bestie dei villani trovate nel castello. E quando i contadini riescono a far uscire le loro bestie di notte dal palazzo e farle sparire, egli ordina di arrestare Migone e gli fa l'interrogatorio. Egli adopera i mezzi tuttora usati dai regimi totalitari, cioè il linguaggio della forza e la tortura, senza ascoltare a Migone che 
gli consiglia di usare la lingua piuttosto che la frusta per capire i problemi dei suoi sudditi:

"Vuoi dire che è tempo perso parlare con te? Forse intendi meglio il linguaggio de la frusta che quello de le parole".

"Vossignoria, vui sete apena apena arivato".

"E allora?"

“Allora vossignoria dovrebbe da parla' massimamente co' le parole. La lingua potrebbe aiutarve mejo che la frusta a capi' li problemi de la gente deqquà".

"A me me interessano le bestie involate dal castello più che i problemi de la gente" (Malerba, 2015, p. 38).

Il marconte non ascolta le parole di nessuno, neanche della moglie, Bernarda, quando gli dice che la sua politica con i sudditi era sbagliata e che doveva imparare a governare, invece di usare sempre la frusta o di aumentare i tributi. Invece di ascoltare il consiglio della moglie, egli decide di raddoppiare i dazi e di costringere i contadini a pagare con la frusta:

"Me doveranno pagare i tributi doppi due volte, sti villani!".

"E tu doverai imparare a governare".

"Governarò a forza di frustate".

"Non bastarà. Governare è arte difficultosa e ardua che se impara per lungo e periglioso esercizio".

"Presto se vedrà come il marconte Berlocchio mettarà a la frusta sti villani despettosi!" (Malerba, 2015, p. 70).

L'autore mette in risalto anche la collaborazione tra il potere politico e quello religioso a scapito del popolo, poiché Belcapo e frate Capuccio si mettono d'accordo per fregare la popolazione. Questo appare chiaro quando il curiale si serve dei registri della parrocchia per fare un elenco dei mestieri e un censimento degli abitanti che dovranno pagare i tributi. Il curiale chiede poi a frate Capuccio di incitare i suoi parrocchiani a digiunare, così si mangia meno e allora avanzerano le vivande per i tributi. Il prete vuole condividere con la corte l'introito, ma il curiale propone solo il decimo delle decime e 
frate Capuccio è costretto ad accettare per evitare lo scontro con il marconte:

"Decima pars de le decime suficere potest. Concordamus?".

"Mica tantum".

"Conviene concordare, frato Capuccio, ad evitandam discussionem con Berlocchio".

A frate Capuccio non garba fare discussioni con il mrconte.

"E concordamus!".

$[\ldots]$.

"Mi raccomando di predicare il digiuno".

"Predicabo degiunum settimanale parziale et domenicale absolutum et totale".

I due si sringono la mano (Malerba, 2015, p. 48).

Con una sfumatura sarcastica Malerba allude alla mediocrità e al fallimento dei regimi totalitari quando il curiale, alla base dei registri della chiesa, compila un elenco approssimativo dei nomi e dei mestieri e comincia a riscuotere $\mathrm{i}$ tributi da vettovaglia per favore della guarnigione. Belcapo corre in piazza, dove la gente è radunata a guardare Migone mentre viene frustato, e comincia a chiamare i nomi del sigillo e con ogni nome la tassa imposta a base del mestiere. Dopo aver pronunciato alcuni nomi e la tassa prestabilita senza ricevere nessuna risposta, il curiale scopre che tutti sono morti e che il registro della parrocchia è rimasto indietro di due o tre generazioni e quelli segnati sono i bisnonni e i trisnonni degli abitanti attuali di Tripalle. Berlocchio allora ordina di fare un censimento nuovo, ma per il momento i discendenti dei defunti devono pagare come i loro avi in moneta sonante. I paesani, però, non hanno le monete e non le hanno mai viste. Il marconte emana allora l'ordinanza che il pagamento dei tributi e delle tasse, per quelli che non hanno monete, avvenga in natura, cioè con i prodotti della terra, della stalla, o del pollaio e quindi vino, olio, grano, salami, prosciutti, pollastri...ecc. I contadini, perciò, si affrettano a nascondere i loro cibi:

"E succede allora che si mettono di lena a scavare buche da tutte le parti. Buche fonde e meno fonde, rotonde o squadrate, larghe o strette, 
bislunghe, a forma di orcio o di cassapanca e qualcuna a forma di presciutto, i fortunati che hanno un presciutto da nascondere. Le buche vengono chiuse con un coperchio di pietra, così che i cani non possono scavare, e nemmeno gli altri animali come le volpi i tassi i gatti selvatici. Un vero cemeterio di roba da magnare" (Malerba, 2015, pp. 69-70).

Un'altra forma di collaborazione tra la chiesa e il governo a scapito del popolo si vede quando il marconte decide di organizzare una grande festa nel cortile del palazzo per derubare i tripallesi. Mentre i villani sono radunati nel cortile a guardare $i$ giochi e $i$ festeggiamenti, il marconte manda un gruppo di soldati con i due armigeri a rastrellare le loro case e confiscare ogni specie di cibo. All'inizio frato Capuccio non si oppone dicendo che si tratta di un latroncinio, ma quando il marconte gli dice che è invece una requisizione, egli benedice l'operazione e chiede il decimo che spetta alla parrocchia:

Frato Capuccio però ritorna sui passi, si avvicina a Berlocchio e gli dice a un orecchio:

"Rammemoro a vossignoria che la decima pars de la requisizione versare debetur pro parocchia".

"Se vedarà".

Frato Capuccio insiste:

"Rammemoro a vossignoria che latronciniun peccatum est".

"Ormai deffinita è stata come requisizione. Tornare indietro non potest".

"Si est requisizione, repeto che la decima pars versare debetur pro parocchia affamata".

"E io non deniego ma repeto che se vedarà" (Malerba, 2015, p. 80).

La lotta prende una nuova svolta quando Berlocchio ordina di catturare Migone, il più colto e il più furbo dei villani, per interrogarlo sulle bestie sparite di notte dal palazzo feudale. Quando il contadino rifiuta di collaborare con il marconte, quest'ultimo ordina di chiuderlo nudo in una gabbia di ferro appesa nel cortile del castello per intimidire tutti i villani del feudo. Per maggiore umiliazione ordina 
anche di coprirlo di merda di bestie, e quando non si trova merda perché non ci sono bestie ordina ai soldati di coprirlo con merda di uomo. Una folla si è radunata a guardare e beffeggiare Migone nudo nella gabbia coperto di Merda, ma lui resiste e cerca di sensibilizzare i villani, per renderli consapevoli della loro realtà:

Ve va de ride, de divertirve, ah! - attacca Migone verso la gente del paese ammucchiata là sotto. - Ve duvrebbe veni' da piagne a tutti quanti invece che da ride! Nun capite o nun ve va de capi' er signifficato der fatto che un tripallese come vui l'hanno dispogliato e smerdato drento la berlina. Nun ve sete manco addomandato er perché de sto fatto. Se vede proprio che nun ve va de fa' un raggionamento perché nun ce sete abituati a raggiona'. Me despiace per vui. Ve duvrebbe basta' de sape' che m'hanno messa qua drento perché nun vulevo racconta' indo' le bestie vostre (Malerba, 2015, pp. 142-143).

Una volta liberato, Migone continua la sua campagna di mobilitazione: comincia a radunare $i$ villani tripallesi e a consapevolizzarli della loro miseria e dell'ingiustizia subita, invitandoli alla vendetta e alla rivoluzione contro l'assolutismo di Berlocchio. Così, pian piano, il popolo di Tripalle si rende conto della sua realtà e comincia ad organizzarsi in una ribellione collettiva che si trasforma in una rivoluzione contro il marconte:

Migone ha radunato nella notte Baldassarre, Galletto, Luigione, Pedrotto e Beletto nel fienile di Baldassarre sotto la ripa dritta. Sono venuti giù dal paese intabarrati nei loro tabarri di lanapecora, neri i tabarri e nere le facce. Una radunata di notte e di soqquatto per parlare, dopo che Migone è stato liberato dalla gabbia e si è lavato da capo a piede. Tutti intorno si siedono i villani e Migone accende un lume e incomincia a dire: "Semo amichi, semo drentro a la merda tutti quanti” (Malerba, 2015, p. 162).

Migone pianifica la propria vendetta, tramando per il marconte una umiliazione più forte di quella subita: mentre Berlocchio e la sua soldataglia fa la guerra con il feudo adiacente, egli entra di nascosto nel castello e ruba un vestito del marconte. Con questo vestito egli addestra un cane a mangiare 1'"uccello" di Berlocchio, riempendo il 
vestito di paglia e mettendo tra le gambe una salsiccia poi lanciando il cane affamato che corre subito a strappare la salsiccia. Abituato il cane a questa azione, Migone comincia a tenere sempre d'occhio il marconte accompagnato dal cane affamato. Un giorno arriva il momento tanto aspettato: durante una partita di caccia e mentre il marconte sta per fare una delle sue porcherie con la figlia della padrona del feudo vicino, e appena slacciati i pantaloni ben memorizzati dal cane, Migone lancia il suo animale che scatta come fulmine strappando i genitali di Berlocchio.

In un crescendo di tensioni negli ultimi tre capitoli del romanzo, lo scontro diventa aperto e irrimediabile, poiché il marconte, invece di rimediare alla situazione e attenuare la rabbia del popolo di Tripalle, manda i suoi soldati a fare incursioni nelle case dei villani e saccheggiare ogni tipo di cibo o di prodotti commestibili:

Che i villani arroganti e strafuttenti vengano puniti con la requisizione de ogni vettovaglia de qualsivoglia tipo se ritrovi riposta ne le case loro. Chi opporrà resistenza che venga prima sfondata la porta con le picche e poi sfondato con le medesime picche anco il villano. E qualora il villano ardisca alzare la mano oltre che la voce, che venga punito con il taglio de l'uccello sul luogo istesso ad evitare che il castello venga imbrattato del sangue loro infetto (Malerba, 2015, p. 225).

Quando poi i villani si raggruppano fuori delle mura del castello e lo assediano facendo strepito e chiedendo la restituzione dei propri beni, il marconte ordina, per l'ennesima volta, di catturare e portare subito Migone. Quest'ultimo viene acchiappato da quattro soldati, mentre sta dormendo nel bosco.

Il confronto tra Migone e Berlocchio, verso la fine del romanzo, è illuminante poiché riassume tutta la filosofia dell'opera. In questo episodio l'autore mette in risalto la crisi politica e lotta di classe tra i villani guidati da Migone da una parte e il marconte e la sua corte dall'altra. La brama di potere di Berlocchio viene contrastata dall'astuzia di Migone che si rivela un uomo molto furbo e tenace, 
consapevole del fatto che un popolo indignato e affamato malgrado sia inerme può diventare tanto pericoloso quando si ribella:

Il marconte diventa tutto rosso nella faccia a sentirsi disconsigliare da un villano.

"I tuoi desconsigli non me interessano!"

"Ve desconsijo per il bene vostro".

"Il bene mio non te deve angustiare!"

"E invece me angustia, vossignoria. Vui duvete sape' che la gente se lagna de brutto, è arrivata a lo stremo de la pazienza. La gente ignorante, li villani come dite vui, quanno se incazzano diventano gattivi, ah!" (Malerba, 2015, p. 234).

Migone dimostra al suo interlocutore la propria efficacia facendo un fischio e allora sul castello comincia una pioggia di sassi lanciati dai suoi compagni, e poi un altro fischio che fa cessare l'attacco. Quando Berlocchio si rende conto della gravità della situazione e della pericolosità di questo villano, cambia strategia cercando di lusingarlo con vari mezzi. Prima lo invita a sedere a tavola con lui e mangiare il coniglio arrosto, poi gli propone di nominarlo cortigiano o di metterlo al comando delle truppe e alla fine anche di dargli un salario annuale e di lasciarlo libero in cambio di tener quieti i villani e di spiarli e maneggiarli per conto del castello. Migone asseconda astutamente il marconte, gli giura di fare tutto questo e prende due monete d'argento come anticipo del salario accordato. Appena uscito dal castello, però, egli va a pianificare l'assedio totale del palazzo con i suoi compagni e racconta loro tutto quello che è successo tra lui e il marconte:

"Ve devo da di' n'antra cosa più importante. Che ciò promesso, a quer zozzone der marconte, che jo faccio le spiate a li danni vostri, che ve persuado de lavura' pe' mntene' lui e quelli antri zozzoni der castello, er curiale, Ulfredo e Manfredo, er frato e tutti li sordati!" (Malerba, 2015, p. 251).

In una scena ispiratrice i tripallesi tengono una specie di tribunale popolare, davanti al palazzo, e prendono alcune decisioni riguardo alla forma del governo dopo la caduta del tiranno e 
condannano alla forca Berlocchio, il curiale Belcapo, i due armigeri Ulfredo e Manfredo e tutti i soldati che oppongono resistenza. Il marconte, da sopra il muraglione sente tutto e si rende conto di essere stato preso in giro da Migone. Le sue decisioni diventano più disastrose: ordina a Ulfredo e Mafredo di prendere i soldati e uscire ad affrontare la popolazione con la forza, ma quando questi rifiutano perché i soldati non sono in grado di farlo perché sono pochi, deboli e non si reggono neanche in piedi dalla fame, egli ordina le sue guardie di appiccare alla forca $i$ due armigeri considerati traditori.

Lo scrittore mette in rilievo il carattere immutevole dei despoti quando Berlocchio, accortosi della sua imminente fine, chiama frato Capuccio per confessargli i propri peccati e chiedergli l'assoluzione. Egli confessa di aver fatto uccidere la moglie, Bernarda, e quando il frate dice di non poter assolverlo dall'uccisione di una persona il Marconte ricorre alle minacce:

"Frato Capuccio, parliamoce chiaro. Io non ho più gnente da perdere, ve faccio mettere a le catene e frustare e anco scorticare la pelle e anco fare i buchi con lo schidone de l'arrosti. Ve conviene darme l'assoluzione e la grazia per il paradiso come ve ho addomandato. Siamo in trappola, frato Capuccio, e conviene che ce aiutiamo in modo vicendevole" (Malerba, 2015, pp. 262-263).

In un'altra scena suggestiva Malerba ci presenta la drammatica fine del tiranno quando, ottenuta l'assoluzione dal parroco, il marconte esce nel cortile dove continuano a piovere i sassi lanciati dai popolani e dice al curiale Belcapo di aver deciso, per non dare soddisfazione ai villani, di appiccarsi insieme a lui alla forca. Appena appiccato Berlocchio alcuni soldati, trascinando i piedi aprono il portone del castello e danno il segno di resa. I villani precipitano dentro il castello dove vedono il marconte e il suo curiale appiccati in mezzo al cortile, mentre frato Capuccio si affaccia da una finestra benedicendo Migone e i suoi “onoratissimi” villani.

Con questa scena Malerba richiama alla mente del lettore scene simili della nostra storia moderna che hanno commemorato la tragica fine di alcuni tiranni come Hitler e Mussolini, Ceausescu e 
Gheddafi...ecc., come se volesse dire che anche se i tempi cambiano il carattere dei despoti e la fine dei regimi totalitari sono sempre gli stessi. Perciò come dice Rinaldo Rinaldi nella prefazione all'opera:

Protagonisti non di una parabola filosofica ma di una lotta di classe senza esclusione di colpi, l'astuto Migone ed i suoi villani riescono a vincere il potere politico, militare e religioso rappresentato da Berlocchio, dai suoi scalcagnati soldati e dall'ipocrita frate Capuccio, proprio perché incarnano il diritto naturale: un diritto indebolito dalla fame e conculcato dalla violenza, ma capace di distruggere i mostri generati dalla sopraffazione (Malerba, 2003, p. VI).

\section{CONCLUSIONE}

Concludendo possiamo dire che l'opera ha un doppio valore poiché, malgrado sia ambientata nel medioevo, può essere considerata la metafora della situazione attuale in diversi Paesi del mondo. Il romanzo, da questo punto di vista, può essere considerato una denuncia di una società in crisi non solo economica, ma anche religiosa e morale, dove mancano i valori e la dignità dell'uomo, e dove domina il male in tutte le sue forme. Questo vuol dire che Malerba era consapevole della situazione politica, economica, religiosa e sociale del proprio Paese, e che il romanzo esprime l'indignazione nutrita dallo scrittore verso tale situazione.

La comicità e l'umorismo che caratterizzano l'opera rappresentano una specie di maschera che cela lo sdegno dello scrittore nei confronti del sistema politico e sociale in Italia degli anni Sessanta e Settanta, ma anche rappresentano una critica verso la realtà politica e sociale alla fine del Novecento in tante altre parti del mondo.

Lo scrittore ha scelto la forma storica per il suo romanzo come mezzo di distanziamento dalla realtà che sta raccontando, cosa che gli consente di penetrare nella percezione del lettore facendolo pensare alla propria realtà, paragonandola a quella di Migone e dei villani nel 
romanzo. Da questo paragone potrebbe scaturire una reazione simile a quella dei cittadini tripallesi.

Con una sfumatura sarcastica l'autore allude al fatto che la dittatura ha come conseguenza la miseria generale e la fame che inducono il popolo alla ribellione e allo scontro con il regime. Egli sottolinea anche che un popolo indignato e affamato, malgrado sia inerme, può diventare tanto pericoloso quando si ribella, e che il carattere dei despoti e la fine dei regimi totalitari sono sempre gli stessi.

L'attualità dell'opera è reflessa dalla sua vicenda che richiama alla mente avvenimenti recenti, tra cui quelli avvenuti dopo la morte dello scrittore, cosa che conferma la natura profetica del romanzo. La vicenda è paragonabile a quello che è successo nello scorso decennio in molti Paesi della primavera araba dove i popoli, per le ingiustizie subite, si sono rivoltati contro i loro governanti. Non solo, ma alla fine dell'opera scaturisce una verità sorprendente, cioè che il cambiamento non migliora sempre la realtà, ma porta spesso nuove dittature più pericolose e più onerose di quelle precedenti.

\section{References}

Barilli, R. (2013), Alla ricerca di un filo conduttore nella narrativa di Malerba, in Catelli, N. (a cura di), Simmetrie naturali, Luigi Malerba tra letteratura e cinema, Parma, Edizioni Diabasis.

Chiafele, A. (2010), Ironia: metafinzione nelle opere di Luigi Malerba, Tesi di dottorato, Graduate Department of Italian Studies, University of Toronto.

Crotti, C. (2016-2017), "Indovina le strutture e $i$ contenuti seguiranno", Tre studi narratologici sul romanzo di Luigi Malerba, Tesi di laurea in Letteratura italiana contemporanea, Università di Bologna, Scuola di Lettere e beni culturali, Anno accademico.

De Palma, R. (2012), Lo scrittore indignato, Stilo editrice, Bari. 
Donati A. (1927) (A cura di), Vittorio Alfieri, Della tirannide e altre opere, Bari, Laterza,

Lenci, L. M. (2013), Contesti Malerbiani, Poetica sperimentale e rappresentazione della scena matrimoniale, Università degli studi di Verona, Scuola di Dottorato di Studi Umanistici, Dipartimento di Culture e Civiltà.

Malerba, L. (2003), ll pataffio, Monte Università Parma Editore, Parma.

Malerba, L. (2015), ll pataffio, Quodlibet, Macerata.

Manacorda, G. (1996), Storia della letteratura italiana contemporanea, Roma, Editori Riuniti.

Mauri, P. (2013), Malerba, storia e geografia in Catelli, N. (a cura di), Simmetrie naturali, Luigi Malerba tra letteratura e cinema, Parma, Edizioni Diabasis. 